\title{
Non-steroidal anti-inflammatory drug use and the risk of benign prostatic hyperplasia-related outcomes and nocturia in the Prostate, Lung, Colorectal, and Ovarian Cancer Screening Trial
}

\author{
Siobhan Sutcliffe ${ }^{\star}$, Robert L. Grubb III ${ }^{\dagger}$, Elizabeth A. Platz ${ }^{\ddagger}$, Lawrence R. Ragard $§$, Thomas \\ L. Riley $\rrbracket$, Sally S. Kazin ${ }^{\dagger \dagger}$, Richard B. Hayes ${ }^{\ddagger \ddagger}$, Ann W. Hsing ${ }^{* \star}$, and Gerald L. Andriole ${ }^{\dagger}$ on \\ behalf of the Urologic Diseases in America Project \\ *Division of Public Health Sciences and the Alvin J. Siteman Cancer Center, Department of \\ Surgery \\ tDivision of Urologic Surgery and the Alvin J. Siteman Cancer Center, Washington University \\ School of Medicine, St Louis, MO \\ ‡Department of Epidemiology, Johns Hopkins Bloomberg School of Public Health, and the Brady \\ Urological Institute and Sidney Kimmel Comprehensive Cancer Center, Johns Hopkins Medical \\ Institutions, Baltimore, MD \\ §Westat, Rockville, MD \\ IInformation Management Services, Rockville, MD \\ ${ }^{* *}$ Division of Cancer Epidemiology and Genetics, National Cancer Institute, National Institutes of \\ Health, Bethesda, MD \\ t'Department of Mathematics, University of Georgia, Athens, GA. \\ \#‡Division of Epidemiology, Department of Environmental Medicine, New York University School \\ of Medicine, New York City, NY, USA
}

\section{Abstract}

- To investigate the relationship between non-steroidal anti-inflammatory drug (NSAID) use and the incidence of benign prostatic hyperplasia (BPH)-related outcomes and nocturia, a lower urinary tract symptom (LUTS) of BPH, in light of accumulating evidence suggesting a role for inflammation in BPH/LUTS development.

- At baseline, participants in the Prostate, Lung, Colorectal, and Ovarian Cancer Screening Trial completed questions on recent, regular aspirin and ibuprofen use, BPH surgery, diagnosis of an enlarged prostate/BPH, and nocturia. Participants in the intervention arm also underwent a digital rectal examination (DRE), from which prostate dimensions were estimated, as well as a prostate-specific antigen (PSA) test. Only participants in the intervention arm without BPH/LUTS at baseline were included in the analysis $(n=$ 4771).

- During follow-up, participants underwent annual DREs and PSA tests, provided annual information on finasteride use, and completed a supplemental questionnaire in 20062008 that included additional questions on diagnosis of an enlarged prostate/BPH and nocturia.

Correspondence to Siobhan Sutcliffe, Division of Public Health Sciences and the Alvin J. Siteman Cancer Center, Department of Surgery, Washington University School of Medicine, 660 South Euclid Avenue, Box 8100, Room 5026, St Louis, MO 63110, USA. sutcliffes@wudosis.wustl.edu. 
- Information collected was used to investigate regular aspirin or ibuprofen use in relation to the incidence of six BPH/LUTS definitions: diagnosis of an enlarged prostate/BPH, nocturia (waking two or more times per night to urinate), finasteride use, any selfreported BPH/LUTS, prostate enlargement (estimated prostate volume $\geq 30 \mathrm{~mL}$ on any follow-up DRE) and elevation in PSA level (> $1.4 \mathrm{ng} / \mathrm{mL}$ on any follow-up PSA test).

- Generally, null results were observed for any recent, regular aspirin or ibuprofen use (risk ratio $=0.92-1.21, P=0.043-0.91$ ) and frequency of use (risk ratio for one category increase in NSAID use $=0.98-1.11, P$-trends $=0.10-0.99)$ with incident BPH/LUTS.

- The findings obtained in the present study do not support a protective role for recent NSAID use in BPH/LUTS development.

\section{Keywords}

aspirin; benign prostatic hyperplasia; ibuprofen; lower urinary tract symptoms; non-steroidal antiinflammatory drugs

\section{Introduction}

$\mathrm{BPH}$ is a common condition of ageing men characterized histologically by hyperplastic nodules in the peri-urethral region and transition zone of the prostate, and clinically by a palpably enlarged prostate and LUTS [1]. Accumulating evidence suggests that inflammation may be involved in BPH/LUTS development [2]. In rodent models, induced prostatic inflammation has been shown to contribute to prostatic hyperplasia [3,4], whereas, in humans, inflammatory infiltrates are frequently observed in prostate tissue specimens from men with BPH [5-8] and the presence or degree of inflammation has been found to be correlated with prostate volume/weight [8,9], LUTS severity [10] and surgery for acute urinary retention independent of urinary tract infection [9]. Finally, in a trial of BPH/LUTS therapy, chronic prostatic inflammation was associated with a greater risk of clinical BPH progression and subsequent acute urinary retention [11].

Given these observations suggesting that inflammation may contribute to BPH/LUTS, it is plausible to hypothesize that anti-inflammatory agents (e.g. NSAIDs) may reduce the risk of BPH/LUTS [12]. To our knowledge, only three previous studies have investigated the association between NSAID use and BPH/LUTS. The first of these studies observed no association between aspirin use and prevalent BPH/LUTS; however, the results were unstable as a result of low numbers of aspirin users [13]. The second study, conducted in the Prostate, Lung, Colorectal and Ovarian (PLCO) Cancer Screening Trial, observed a weak positive association between regular NSAID use and prevalent BPH/LUTS [14], whereas the third study, conducted in the Olmsted County Study of Urinary Symptoms and Health Status among Men, observed a strong inverse association between daily NSAID use and incident BPH/LUTS [12]. Given these strong latter findings for risk (as opposed to the prevalence) of BPH/LUTS, we have now extended the previous analysis in the PLCO to investigate NSAID use in relation to risk of BPH/LUTS.

\section{Patients and methods}

\section{Study population and design}

The PLCO is a large, ongoing randomized controlled trial designed to investigate the effects of prostate, lung, colorectal and ovarian cancer screening on cancer-specific mortality [15]. All men aged 55-74 years with no reported histories of prostate cancer or radical prostatectomy and no reported use of 5a-reductase inhibitors (e.g. finasteride) in the previous 6 months were eligible for the trial. From 1993 to 2001, 76705 men were recruited 
at 10 screening centres across the USA. Half of these participants were randomized to the intervention arm, which included four annual DREs and six annual PSA tests, and half were randomized to the control arm, which consisted of routine medical care. At baseline, participants completed an epidemiological questionnaire, which included questions on NSAID use and BPH/LUTS. Participants in the intervention arm also completed a baseline food frequency and physical activity questionnaire. Every year subsequent to baseline, participants have completed brief annual study update questionnaires to revise their cancer information and provide information on finasteride use. Participants also completed a supplemental epidemiological questionnaire in 2006-2008, including additional questions on NSAID use and BPH/LUTS.

The present analysis was limited to men in the intervention arm $(n=38340)$ to include DRE and PSA information on all men. We further excluded participants who: (i) reported a history of cancer (except non-melanoma skin cancer) at baseline $(n=827)$; (ii) were diagnosed with prostate cancer before completion of the supplemental questionnaire because prostate cancer or its treatment may alter the risk of some BPH/LUTS definitions (e.g. nocturia, $n=2663$ ); (iii) died before the supplemental questionnaire was mailed ( $n=4220$ ); (iv) did not complete the baseline $(n=887)$ or supplemental questionnaires $(n=7467)$ or provided incomplete information on BPH/LUTS $(n=1203)$ or NSAID use $(n=78)$ on these questionnaires; (v) missed or had invalid baseline $(n=1097)$ or follow-up DRE and PSA test results $(n=145)$; and (vi) did not complete the baseline food frequency and physical activity questionnaire $(n=1867)$, leaving 17886 potential participants for analysis. Men excluded because of missing information were less likely to be white, highly educated and married, and slightly more likely to have comorbidities.

Because the focus of the present analysis was BPH/LUTS risk, we further excluded men with any suggestion of BPH/LUTS at baseline. We determined BPH/LUTS at baseline using information obtained from the baseline questionnaire, as well as the baseline DRE and PSA tests. There were three questions related to BPH/LUTS included on the baseline questionnaire: one on a history of surgical procedures of the prostate, including TURP and prostatectomy for benign disease; one on a history of a physician diagnosis of an enlarged prostate/BPH; and another on typical frequency of waking during the night to urinate in the past year (i.e. nocturia). This latter symptom is one of seven LUTS collected on the IPSS (previously known as the AUA Symptom Index) used to assess the severity of BPH-related LUTS [16]. For each DRE, transverse (width) and sagittal (length) prostate dimensions were estimated by palpation in $0.5-\mathrm{cm}$ increments, and used to calculate prostate volume with the formula: volume $=(\pi / 6) \times$ width $^{2} \times$ length [17]. To reduce measurement error, only values from examiners who had performed at least 100 DREs were included and volumes were corrected for examiner bias (i.e. some examiners consistently estimate higher or lower than others) by subtracting the difference between examiner-specific mean volumes and the total mean volume from each value. These steps were found to reduce measurement error in a previous analysis of prostate volume in the PLCO [17]. Finally, with respect to PSA, PSA levels were recorded for each PSA test. Although the PSA level is influenced by other conditions, such as prostatitis, it is reported to be a strong predictor of BPH/LUTS in men without prostate cancer [18-20].

For the present analysis, we excluded men who reported: (i) BPH surgery ( $n=633)$; (ii) diagnosis of an enlarged prostate/BPH $(n=2784)$; or (iii) nocturia $(n=3790)$ (i.e. waking two or more times per night to urinate) [21] on the baseline questionnaire; as well as men with evidence of (iv) prostate enlargement $(n=4208)$ (i.e. estimated prostate volume $\geq 30$ $\mathrm{mL})$ [22] on their baseline DRE or (v) elevation in PSA level $(n=1700)$ (i.e. PSA level > $1.4 \mathrm{ng} / \mathrm{mL}$ ) [12] on their baseline PSA test, leaving $4771 \mathrm{men}$ in the analytic cohort. This number of exclusions was expected based on the older age at entry into PLCO (i.e. 55-74 
years). We also performed BPH/LUTS measure-specific sensitivity analyses excluding only men with the BPH/LUTS measure of interest and men with a history of BPH surgery at baseline, obtaining similar results.

\section{Exposure assessment}

Any 'regular' use and frequency of use of aspirin or aspirin-containing products, such as Bayer, Bufferin or Anacin; and ibuprofen-containing products, such as Advil, Nuprin or Motrin, in the past year were ascertained on the baseline questionnaire. We used this information to create three exposure variables each for aspirin and ibuprofen use: (i) any use; (ii) frequency of use; and (iii) at least daily vs non-daily use (including no use) for comparison with findings from the Olmsted County Study [12]. Similar questions were asked on the supplemental questionnaire and were used to create variables for consistent aspirin and ibuprofen use during follow-up, or at least in the years preceding the baseline and supplemental questionnaires. Finally, on the supplemental questionnaire, participants were also asked for how many years they had taken aspirin or non-aspirin NSAIDs, including ibuprofen, at least once per week. We used this question to create variables for the duration of aspirin and non-aspirin NSAID use for investigation in relation to prevalent BPH/LUTS. No questions on indication for NSAID use were included in the PLCO questionnaires.

\section{Outcome assessment}

There were two questions related to BPH asked on the supplemental questionnaire: one on a history of a physician diagnosis of an enlarged prostate/BPH and one on typical frequency of waking during the night to urinate in the past year. Participants were also asked about the use of finasteride (Proscar or Propecia) in the past year on each annual study update. We used these questions to define incident diagnosis of an enlarged prostate/BPH as an affirmative response to the diagnosis question and incident nocturia as regularly waking two or more times per night to urinate in the past year [21]. This latter definition was limited to men with no reported use of finasteride because this drug may influence nocturia. We defined incident finasteride use as any reported finasteride use before completion of the supplemental questionnaire. Because finasteride can be used for both BPH (Proscar) and hair loss (Propecia), we performed sensitivity analyses limiting the finasteride definition to men who also reported a diagnosis of an enlarged prostate/BPH with similar results. Finally, we combined information from all questions into one self-reported BPH/LUTS endpoint.

With respect to measured BPH-related outcomes, incident prostate enlargement was defined as an estimated prostate volume $230 \mathrm{~mL}$ on any follow-up DRE [22] and incident PSA elevation as a PSA level $>1.4 \mathrm{ng} / \mathrm{mL}$ on any follow-up PSA test [12]. We limited these definitions to men who did not report finasteride use because this drug may influence prostate size and PSA. Each of the above-described definitions captures one or more aspects of $\mathrm{BPH} / \mathrm{LUTS}$.

\section{Statistical analysis}

To begin to investigate confounding, we calculated age-adjusted means and proportions of potential confounding variables by frequency of aspirin and ibuprofen use using linear regression. Variables investigated as confounders were race, education, marital status, body mass index $\left(\mathrm{kg} / \mathrm{m}^{2}\right)$, physical activity, smoking history, consumption of total energy, carbohydrates, fats, polyunsaturated fats, proteins, alcohol, fruit, vegetables, red meat, antioxidant nutrients (i.e. $\beta$-carotene, selenium, vitamins $\mathrm{A}, \mathrm{C}$ and $\mathrm{E}$, as well as zinc, from the diet and supplements, and dietary $a$-carotene, $\beta$-cryptoxanthin, lutein, zeaxanthin and lycopene), multivitamin use and histories of other medical conditions (i.e. hypertension, coronary heart disease [CHD], stroke, diabetes, arthritis, colon polyps and polyp syndromes 
and clinical prostatitis). We investigated associations between NSAID use and incidence or prevalence of BPH/LUTS by calculating risk ratios (RRs) and prevalence ratios, respectively, using Poisson regression with robust variance estimation [23]. We further investigated confounding by: (i) adding each potential confounding variable individually to the regression model and evaluating its influence on the point estimates for aspirin and ibuprofen use; (ii) mutually adjusting for aspirin and ibuprofen use; and (iii) restricting the analyses to men without comorbidities for which NSAIDs are indicated/associated and that may be statistically associated with BPH/LUTS (i.e. histories of hypertension, CHD, stroke, diabetes, arthritis and clinical prostatitis). For the first strategy, any variables found to shift the point estimates for aspirin or ibuprofen use with any BPH/LUTS endpoint were retained in all models. Finally, we performed stratified analyses by age $(<60$ years vs $\geq 60$ years of age) and time between completion of the baseline and supplemental questionnaires ( $<10 \mathrm{vs}$ $\geq 10$ years).

\section{Results}

Of the 4771 eligible participants, 50.9\% reported any regular aspirin use in the past year and $27.4 \%$ reported at least daily use. For ibuprofen, these percentages were $25.3 \%$ and $7.0 \%$, respectively. Some $15.9 \%$ of men reported both aspirin and ibuprofen use. Men who reported a greater frequency of aspirin or ibuprofen use were less likely to be Asian or Pacific Islanders, and more likely to be obese (body mass index $\geq 30 \mathrm{~kg} / \mathrm{m}^{2}$ ), to consume greater amounts of most food groups and nutrients examined, and to have histories of hypertension, CHD and stroke (in the case of aspirin), as well as arthritis and possibly diabetes and prostatitis (Table 1). Participants were followed for a median (range) of 9 (513) years for BPH/LUTS development. By the date of the supplemental questionnaire, 1461 $(30.6 \%)$ reported an incident diagnosis of an enlarged prostate/BPH, 1536 (32.2\%) reported nocturia, 142 (3.0\%) reported finasteride use, 2527 (53.0\%) had had a prostate volume measurement $\geq 30 \mathrm{~mL}$, and $1335(28.0 \%$ ) had had a PSA level $>1.4 \mathrm{ng} / \mathrm{mL}$ (categories are not mutually exclusive). Age-specific incidence rates are provided in the Appendix (Table A1).

\section{Any regular NSAID use in the year before baseline}

No associations were observed between any regular aspirin use and the risk of a diagnosis of an enlarged prostate/BPH or nocturia defined as regularly waking two or more times per night (Table 2). For ibuprofen, a weak, non-statistically significant positive association was observed for a physician diagnosis, whereas a weak, non-statistically significant inverse association was observed for nocturia. When nocturia was defined as regularly waking three or more times per night, a modest positive association was observed for aspirin use in minimally adjusted analyses (relative risk, RR, 1.23 ; 95\% confidence interval, CI, 1.021.49) that attenuated slightly after adjustment for a history of hypertension (multivariableadjusted RR, 1.18; 95\% CI, 0.97-1.44); no association was observed for ibuprofen use (multivariable-adjusted RR, 1.03; 95\% CI, 0.83-1.29). Although not statistically significant and based on smaller numbers, a suggestion of a positive association was observed between aspirin use and the risk of finasteride use, although not between ibuprofen and finasteride use. When information for all self-reported endpoints was combined, a modest positive association was observed between aspirin use and the risk of any self-reported BPH-related outcomes in minimally adjusted analyses; this association attenuated after adjustment for comorbidities and diet. No association was observed for ibuprofen use. With respect to evidence of prostate enlargement on the PLCO-administered DRE, aspirin use was associated with a modestly lower risk of prostate enlargement, particularly after adjustment for a history of CHD and race, whereas no association was observed for ibuprofen use. Generally, similar inferences were obtained in sensitivity analyses defining prostate 
enlargement as: (i) prostate volume $\geq 30 \mathrm{~mL}$ on at least two follow-up or two consecutive follow-up DREs; (ii) uncorrected prostate volume $\geq 30 \mathrm{~mL}$ on at least one or two follow-up DREs; and (iii) examiner-noted prostate enlargement on at least one or two follow-up DREs, with the exception of a weak positive association between ibuprofen use and prostate enlargement defined as examiner-noted enlargement (RR, 1.08; 95\% CI, 1.00-1.18). Finally, with respect to PSA level, no association was observed for aspirin or ibuprofen use. Similar null results were also observed in sensitivity analyses defining PSA elevation as: (i) a follow-up PSA level $>1.5 \mathrm{ng} / \mathrm{mL}$; (ii) at least two follow-up or two consecutive follow-up PSA levels $>1.4 \mathrm{ng} / \mathrm{mL}$ or $>1.5 \mathrm{ng} / \mathrm{mL}$; and (iii) a large change in PSA over follow-up (PSA slope $>$ 80th percentile) [12].

\section{Frequency of regular NSAID use in the year before baseline}

Considering the frequency of NSAID use, no trend was observed between an increasing frequency of regular aspirin use and the risk of a diagnosis of an enlarged prostate/BPH (Table 3). For ibuprofen use, modest positive associations were observed for lower frequencies of ibuprofen use, although not for the highest frequency of use (two or more per day), resulting in a null trend. This pattern also explained the modest, non-significant positive association observed for any ibuprofen use and the risk of a diagnosis of an enlarged prostate/BPH in Table 2. For nocturia, a weak positive trend was observed with an increasing frequency of aspirin use in minimally adjusted analyses. However, this trend was driven solely by positive findings for the greatest category of aspirin use and attenuated after multivariable-adjustment, particularly for a history of hypertension. When nocturia was defined as regularly waking three or more times per night to urinate, a pattern more consistent with a positive dose-response was observed, although this trend was not statistically significant (multivariable-adjusted RR for one category increase in frequency of use, $1.04 ; 95 \%$ CI, 0.99-1.10). For ibuprofen, no trend was observed with nocturia defined as regularly waking two or more times per night, whereas a modest, non-significant positive trend was observed when nocturia was defined as regularly waking three or more times per night (RR, 1.05; 95\% CI, 0.98-1.12). For finasteride use, a non-significant positive trend was observed with increasing frequency of regular aspirin but not ibuprofen use. Finally, when all self-reported information was combined, a weak, non-significant positive trend was observed for aspirin use in minimally adjusted analyses that attenuated after multivariable adjustment, particularly for a history of hypertension. No trends were observed for ibuprofen use.

With respect to prostate enlargement, whereas no trend was observed with an increasing frequency of aspirin use in minimally adjusted analyses, a weak non-significant inverse trend was observed after multivariable adjustment, particularly for a history of CHD and race. However, multivariable-adjusted inverse associations were limited to lower frequencies of aspirin use. No trend was observed between ibuprofen use and the risk of prostate enlargement in minimally or more extensively adjusted analyses. Generally, similar results were observed in sensitivity analyses using the alternate prostate enlargement definitions described above, with the exception of analyses defining prostate enlargement as examiner-noted prostate enlargement; for that endpoint, a positive trend was observed with increasing ibuprofen use (RR, 1.03; 95\% CI, 1.01-1.06). Finally, no trends were observed between an increasing frequency of aspirin or ibuprofen use and the risk of PSA elevation by all definitions considered (Table 3 ).

\section{Duration of regular NSAID use}

No trends were observed between an increasing duration of aspirin or non-aspirin NSAID use and the prevalence of both a diagnosis of an enlarged prostate/BPH and finasteride use (Table 4). For nocturia, weak positive trends were observed with an increasing duration of 
aspirin and non-aspirin NSAID use in minimally adjusted analyses; these positive trends attenuated slightly for aspirin after adjustment for a history of hypertension, and attenuated completely for non-aspirin NSAID use after adjustment for a history of arthritis and body mass index. No trends were observed when nocturia was defined as waking three or more times per night (multivariable-adjusted RR for each 10-year increase in duration of aspirin use, 1.03; 95\% CI, 0.92-1.15; ibuprofen use: RR, 1.08; 95\% CI, 0.92-1.27). A weak, nonsignificant positive trend was observed for aspirin use and the prevalence of any selfreported BPH/LUTS but not for non-aspirin NSAID use. For prostate enlargement, although no trend was observed with an increasing duration of aspirin use in minimally adjusted analyses, a weak inverse trend was observed after adjustment for a history of CHD and race. Finally, no trends were observed for non-aspirin NSAID use with prevalent prostate enlargement, nor for aspirin and non-aspirin NSAID use with prevalent PSA elevation. Generally, similar results were observed in sensitivity analyses using alternate prostate enlargement and PSA elevation definitions, with the exception of analyses defining prostate enlargement as examiner-noted enlargement; in those analyses, no trend was observed with increasing frequency of aspirin use (RR, 0.99; 95\% CI, 0.95-1.04).

In all analyses, similar inferences were obtained after mutual adjustment for aspirin and ibuprofen or non-aspirin NSAID use, and after restriction to men without comorbidities, with the exception of results for nocturia; for this endpoint, associations attenuated towards the null or became weakly inverse among men without comorbidities. No discernable patterns were observed after stratification by age ( $<60$ years vs $\geq 60$ years) and follow-up time ( $<10$ years vs $\geq 10$ years). The results tended to be slightly stronger when the analyses were limited to men who reported consistent aspirin or ibuprofen use on the baseline and supplemental questionnaires (data not shown).

\section{Discussion}

In this prospective analysis of NSAID use and BPH/LUTS, null results were generally observed for recent, regular aspirin and ibuprofen use with the risk of several BPH-related outcomes, and for the duration of regular aspirin and non-aspirin NSAID use with prevalent BPH/LUTS. Possible exceptions to these statements were weak, non-statistically significant positive associations for aspirin use with the risk of nocturia defined more stringently as regularly waking three or more times per night to urinate and finasteride use, and for a longer duration of aspirin and non-aspirin NSAID use with the prevalence of nocturia defined as regularly waking two or more times per night. Weak inverse associations were also observed for aspirin use and the risk of prostate enlargement defined by estimated prostate volume, and for a longer duration of aspirin use and the prevalence of prostate enlargement.

Our generally null findings for recent, regular NSAID use and BPH/LUTS risk differ from those from the only other study [12], to our knowledge, investigating NSAID use and the risk of BPH/LUTS. That study, conducted among participants in the Olmsted County Study, observed strong inverse associations between current, daily NSAID use (either aspirin or non-aspirin NSAIDs) and the risk of several measures of BPH/LUTS, including moderate/ severe LUTS, nocturia defined as regularly waking two or more times per night, low maximum flow rate, prostate volume $>30 \mathrm{~mL}$ as determined by transrectal ultrasonography, PSA level > $1.4 \mathrm{ng} / \mathrm{mL}$ and treatment for BPH [12]. Although information for some of these $\mathrm{BPH} / \mathrm{LUTS}$ definitions was not collected in the PLCO to allow direct comparisons between the two studies, where the definitions of the present study overlapped (i.e. nocturia, PSA level $>1.4 \mathrm{ng} / \mathrm{mL}$, and possibly prostate volume $>30 \mathrm{~mL}$, and BPH treatment), no associations were still observed. No associations were also observed when an exposure definition was used (i.e. daily vs non-daily NSAID use) and study design (i.e. BPH/LUTS 
measure-specific analyses excluding only men with the BPH/LUTS measure of interest at baseline) were used similar to that in the Olmsted County Study. Finally, although the age distribution differed between the present study population (age 55-74 years) and the Olmsted County study population (age 40-79 years), these differences are unlikely to explain the differences in study findings because stronger results were generally observed in older Olmsted County study participants who were closer in age to the PLCO participants. Thus, the reasons for differing findings are unclear and may simply reflect chance or possibly the differing patterns of long-term NSAID use or differing indications for use in the two cohorts not captured by the study questionnaires. For example, age-specific prevalences of daily NSAID use were considerably higher among participants in the Olmsted County study than for PLCO participants, perhaps suggesting differing patterns or indications for use.

Although the findings of the present study were generally null, some weak, marginally significant associations were observed for certain BPH/LUTS endpoints. These findings were more consistent with the results from the previous investigation of NSAID use and prevalent BPH/LUTS in the PLCO, in which modest, although statistically significant, positive associations were observed for both recent, regular aspirin and ibuprofen use with a prevalent diagnosis of an enlarged prostate/BPH, nocturia defined as regularly waking two or more times per night and BPH surgery [14]. We consider that the weakly positive findings of the present study likely represent residual confounding by indication because our findings attenuated towards the null: (i) when we adjusted for markers of NSAID indication on the baseline questionnaire (histories of comorbidities), suggesting that they may have attenuated even further with additional, more detailed comorbidity data or with information on indication for use, and (ii) when we restricted the analyses to men without comorbidities. Further reasons for our weak, marginally significant findings are the large sample size of our analysis, making very weak findings that would not be detected in smaller studies marginally statistically significant in our study (80\% post-hoc power to detect RRs as small as 0.92 ), as well as the possibility of chance. This may be especially true for weak inverse associations observed solely for aspirin use and prostate enlargement.

In conclusion, our generally null findings for NSAID use and incident BPH/LUTS do not support a protective role for recent NSAID use in BPH/LUTS development. Although the findings of the present study with respect to a longer duration of NSAID use and BPH/ LUTS prevalence are also not supportive, additional studies examining the duration of NSAID use in relation to BPH/LUTS incidence are needed to more thoroughly address this question. Moreover, because the preset study and the Olmsted County Study [12] are the only two studies, to our knowledge, investigating NSAID use in relation to incident BPH/ LUTS, additional large studies of this possible association are warranted, particularly with more detailed NSAID exposure information (e.g. duration, dose and indication for use) before any conclusions can be made.

\section{Acknowledgments}

This analysis was funded by the Urologic Diseases in America Project (N01-DK-7-0003). The authors thank Drs Paul Pinsky (National Cancer Institute) and Jennifer St Sauver (Mayo Clinic College of Medicine) for helpful discussion relating to the manuscript, as well as Drs Christine Berg and Philip Prorok (Division of Cancer Prevention, National Cancer Institute), the Screening Center investigators and staff of PLCO, the staff at Information Management Services Inc., and Barbara O'Brien and staff (Westat) for their contributions to PLCO.

\section{Abbreviations}

PLCO Prostate, Lung, Colorectal and Ovarian 


\section{References}

1. Roehrborn, CG.; McConnell, JD. Benign prostatic hyperplasia: etiology, pathophysiology, epidemiology, and natural historyeds. In: Wein, AJ.; Kavoussi, LR.; Novick, AC.; Partin, AW.; Peters, CA., editors. Campbell-Walsh Urology. 9th edn. Vol. Vol. 3. Chapt 86. Philadelphia, PA: Saunders Elsevier; 2007. p. 2649-2673.

2. Nickel JC. Inflammation and benign prostatic hyperplasia. Urol Clin North Am. 2008; 35:109-115. [PubMed: 18061029]

3. Kessler OJ, Keisari Y, Servadio C, Abramovici A. Role of chronic inflammation in the promotion of prostatic hyperplasia in rats. J Urol. 1998; 159:1049-1053. [PubMed: 9474230]

4. Robinette CL. Sex-hormone-induced inflammation and fibromuscular proliferation in the rat lateral prostate. Prostate. 1988; 12:271-286. [PubMed: 2453862]

5. Odunjo EO, Elebute EA. Chronic prostatitis in benign prostatic hyperplasia. Br J Urol. 1971; 43:333-337. [PubMed: 4104172]

6. Nickel JC, Downey J, Young I, Boag S. Asymptomatic inflammation and/or infection in benign prostatic hyperplasia. BJU Int. 1999; 84:976-981. [PubMed: 10571623]

7. Kohnen PW, Drach GW. Patterns of inflammation in prostatic hyperplasia: a histologic and bacteriologic study. J Urol. 1979; 121:755-760. [PubMed: 88527]

8. Di Silverio F, Gentile V, De Matteis A, et al. Distribution of inflammation, pre-malignant lesions, incidental carcinoma in histologically confirmed benign prostatic hyperplasia: a retrospective analysis. Eur Urol. 2003; 43:164-175. [PubMed: 12565775]

9. Mishra VC, Allen DJ, Nicolaou C, et al. Does intraprostatic inflammation have a role in the pathogenesis and progression of benign prostatic hyperplasia? BJU Int. 2007; 100:327-331. [PubMed: 17617139]

10. Nickel JC, Roehrborn CG, O'Leary MP, Bostwick DG, Somerville MC, Rittmaster RS. The relationship between prostate inflammation and lower urinary tract symptoms: examination of baseline data from the REDUCE trial. Eur Urol. 2008; 54:1379-1384. [PubMed: 18036719]

11. Roehrborn CG. Definition of at-risk patients: baseline variables. BJU Int. 2006; 97(Suppl. 2):7-11. [PubMed: 16507046]

12. St Sauver JL, Jacobson DJ, McGree ME, Lieber MM, Jacobsen SJ. Protective association between nonsteroidal antiinflammatory drug use and measures of benign prostatic hyperplasia. Am J Epidemiol. 2006; 164:760-768. [PubMed: 16905643]

13. Meigs JB, Mohr B, Barry MJ, Collins MM, McKinlay JB. Risk factors for clinical benign prostatic hyperplasia in a community-based population of healthy aging men. J Clin Epidemiol. 2001; 54:935-944. [PubMed: 11520654]

14. Kang D, Andriole GL, Van De Vooren RC, et al. Risk behaviours and benign prostatic hyperplasia. BJU Int. 2004; 93:1241-1245. [PubMed: 15180615]

15. Prorok PC, Andriole GL, Bresalier RS, et al. Design of the Prostate, Lung, Colorectal and Ovarian (PLCO) Cancer Screening Trial. Control Clin Trials. 2000; 21:273S-309S. [PubMed: 11189684]

16. Barry MJ, Fowler FJ Jr, O'Leary MP, et al. The American Urological Association symptom index for benign prostatic hyperplasia. The Measurement Committee of the American Urological Association. J Urol. 1992; 148:1549-1557. [PubMed: 1279218]

17. Pinsky PF, Kramer BS, Crawford ED, et al. Prostate volume and prostate-specific antigen levels in men enrolled in a large screening trial. Urology. 2006; 68:352-356. [PubMed: 16904451]

18. Roehrborn CG, McConnell JD, Lieber M, et al. Serum prostate-specific antigen concentration is a powerful predictor of acute urinary retention and need for surgery in men with clinical benign prostatic hyperplasia. PLESS Study Group. Urology. 1999; 53:473-480. [PubMed: 10096369]

19. Roehrborn CG, McConnell J, Bonilla J, et al. Serum prostate specific antigen is a strong predictor of future prostate growth in men with benign prostatic hyperplasia. PROSCAR longterm efficacy and safety study. J Urol. 2000; 163:13-20. [PubMed: 10604304]

20. Vesely S, Knutson T, Damber JE, Dicuio M, Dahlstrand C. Relationship between age, prostate volume, prostate-specific antigen, symptom score and uroflowmetry in men with lower urinary tract symptoms. Scand J Urol Nephrol. 2003; 37:322-328. [PubMed: 12944191] 
21. Abrams P. Nocturia: the major problem in patients with lower urinary tract symptoms suggestive of benign prostatic obstruction (LUTS/BPO). Eur Urol Suppl. 2005; 3:8-16.

22. Siami P, Roehrborn CG, Barkin J, et al. Combination therapy with dutasteride and tamsulosin in men with moderate-to-severe benign prostatic hyperplasia and prostate enlargement: the CombAT (Combination of Avodart and Tamsulosin) trial rationale and study design. Contemp Clin Trials. 2007; 28:770-779. [PubMed: 17761460]

23. Spiegelman D, Hertzmark E. Easy SAS calculations for risk or prevalence ratios and differences. Am J Epidemiol. 2005; 162:199-200. [PubMed: 15987728] 


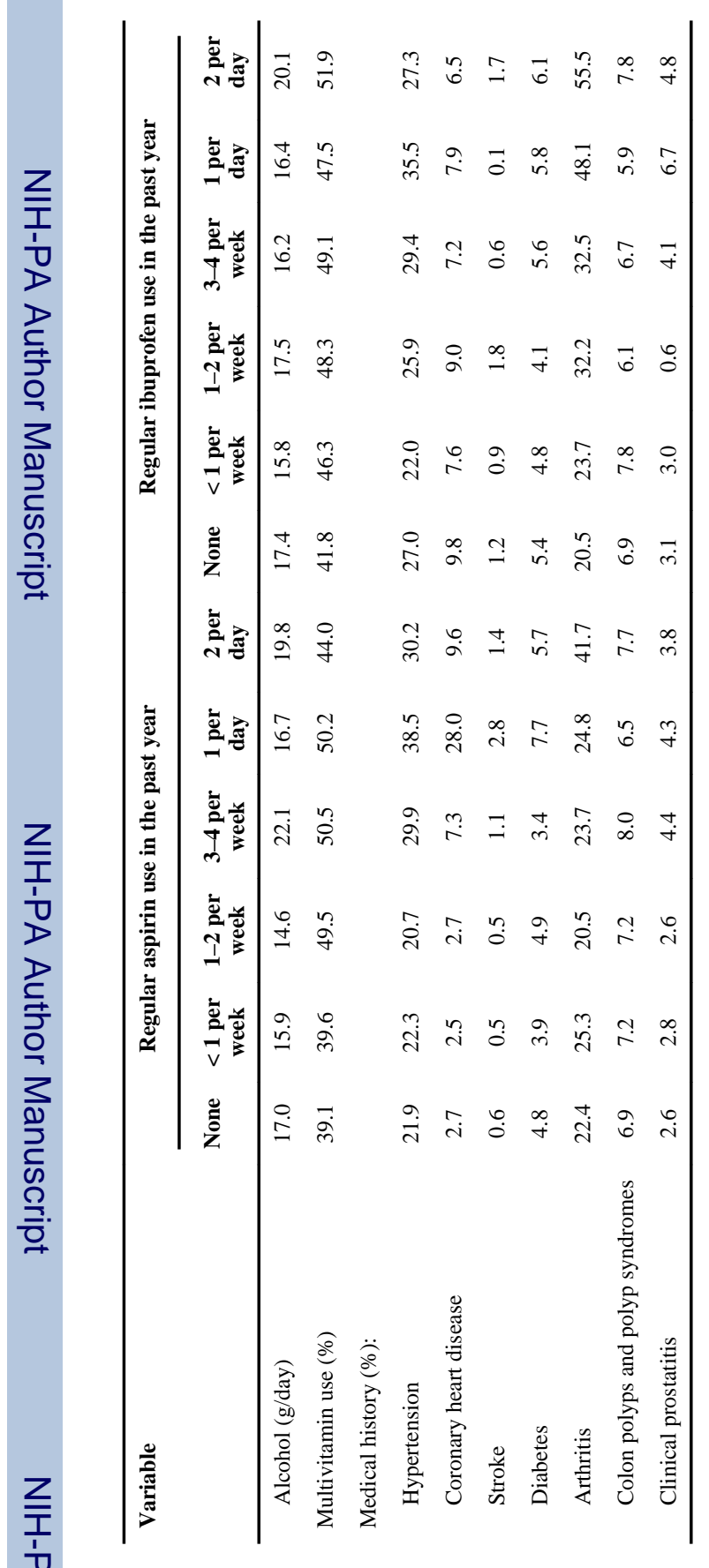

BJU Int. Author manuscript; available in PMC 2013 October 01. 


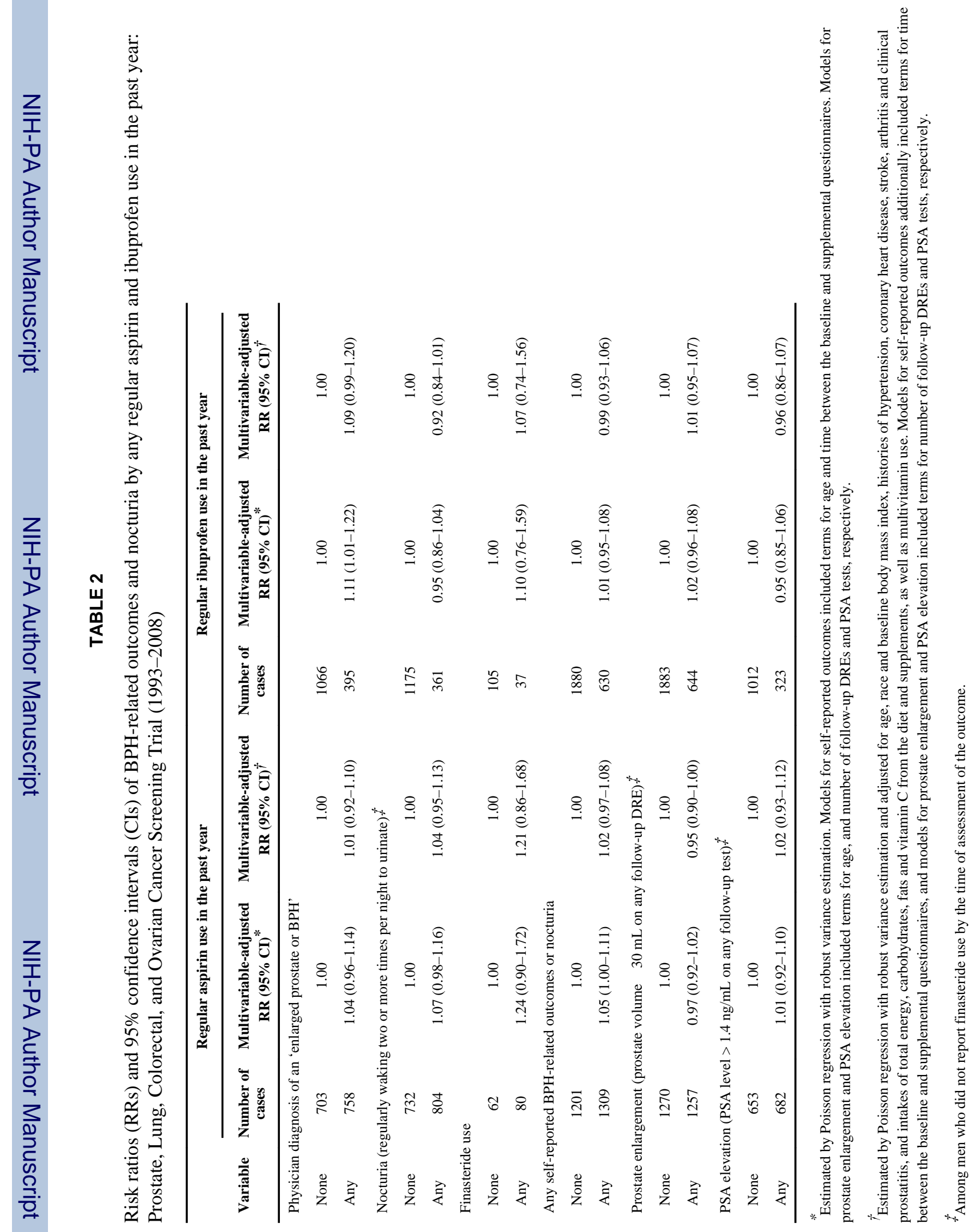




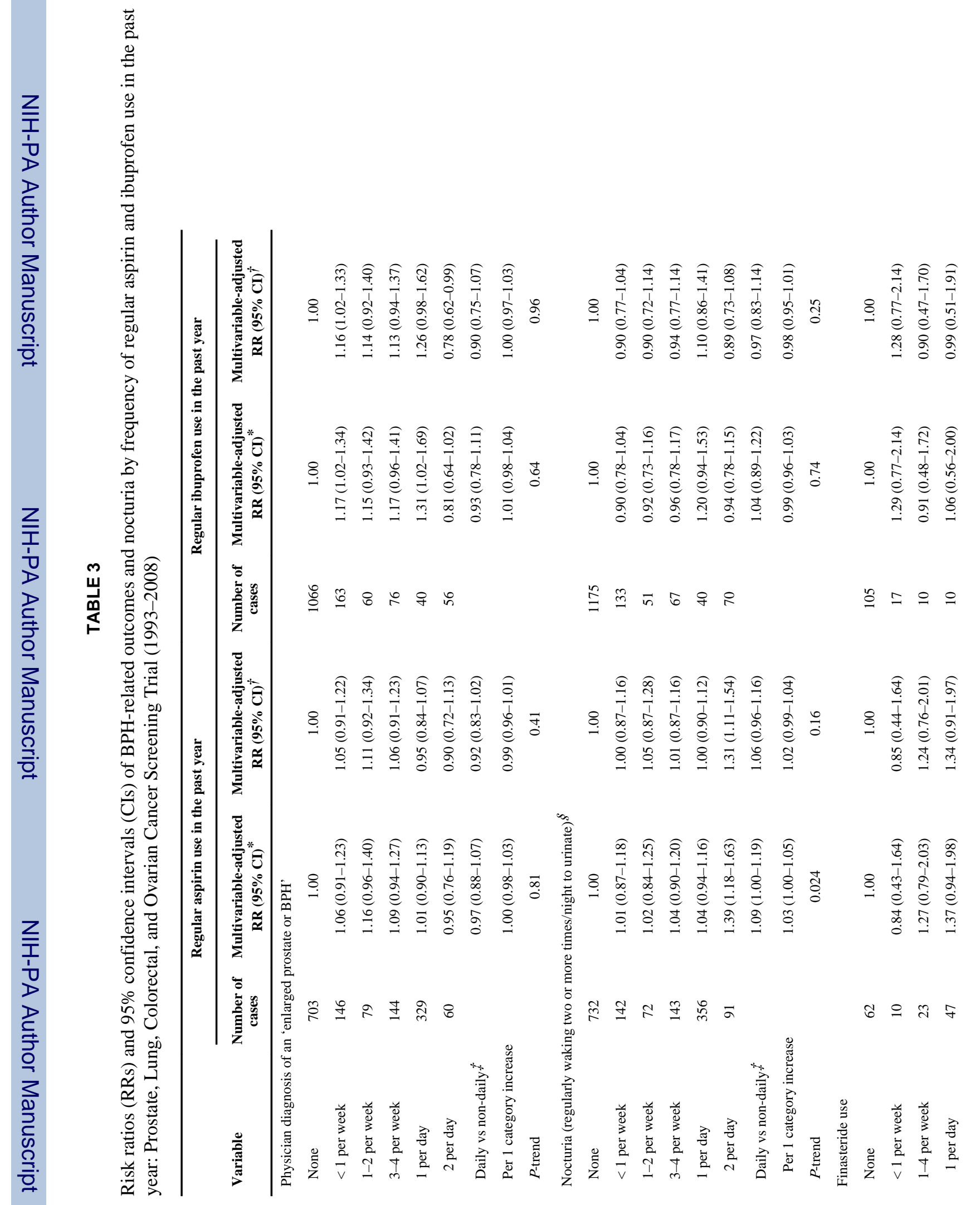

BJU Int. Author manuscript; available in PMC 2013 October 01. 


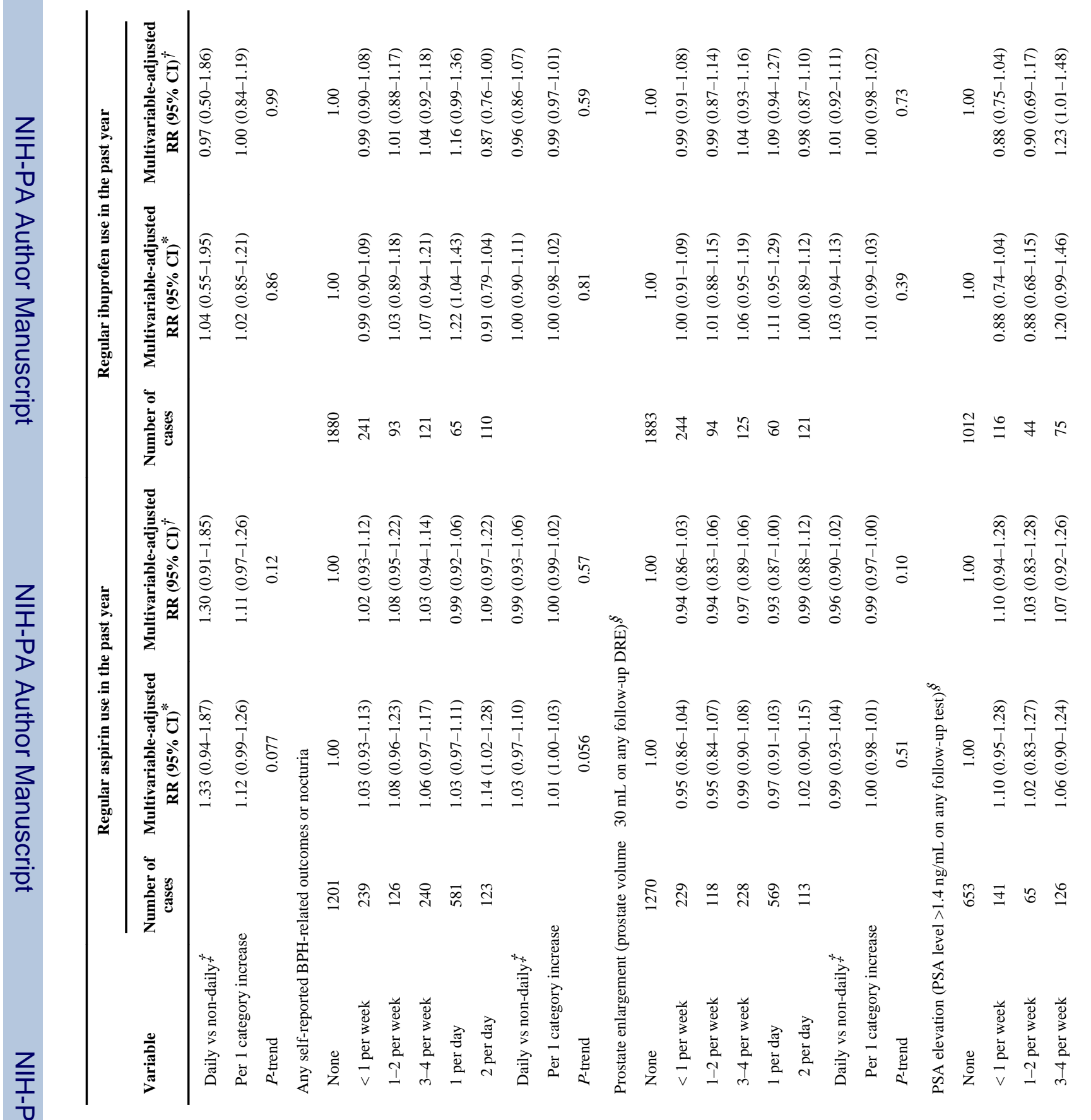

BJU Int. Author manuscript; available in PMC 2013 October 01. 


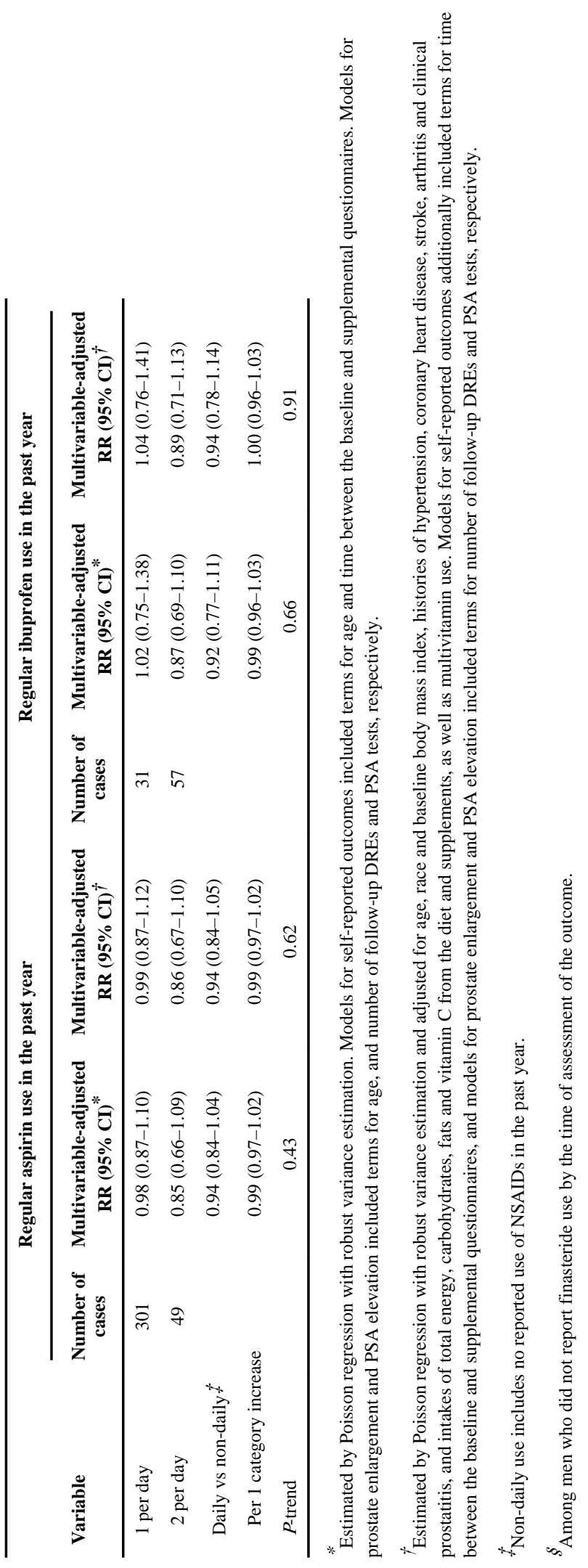

BJU Int. Author manuscript; available in PMC 2013 October 01. 


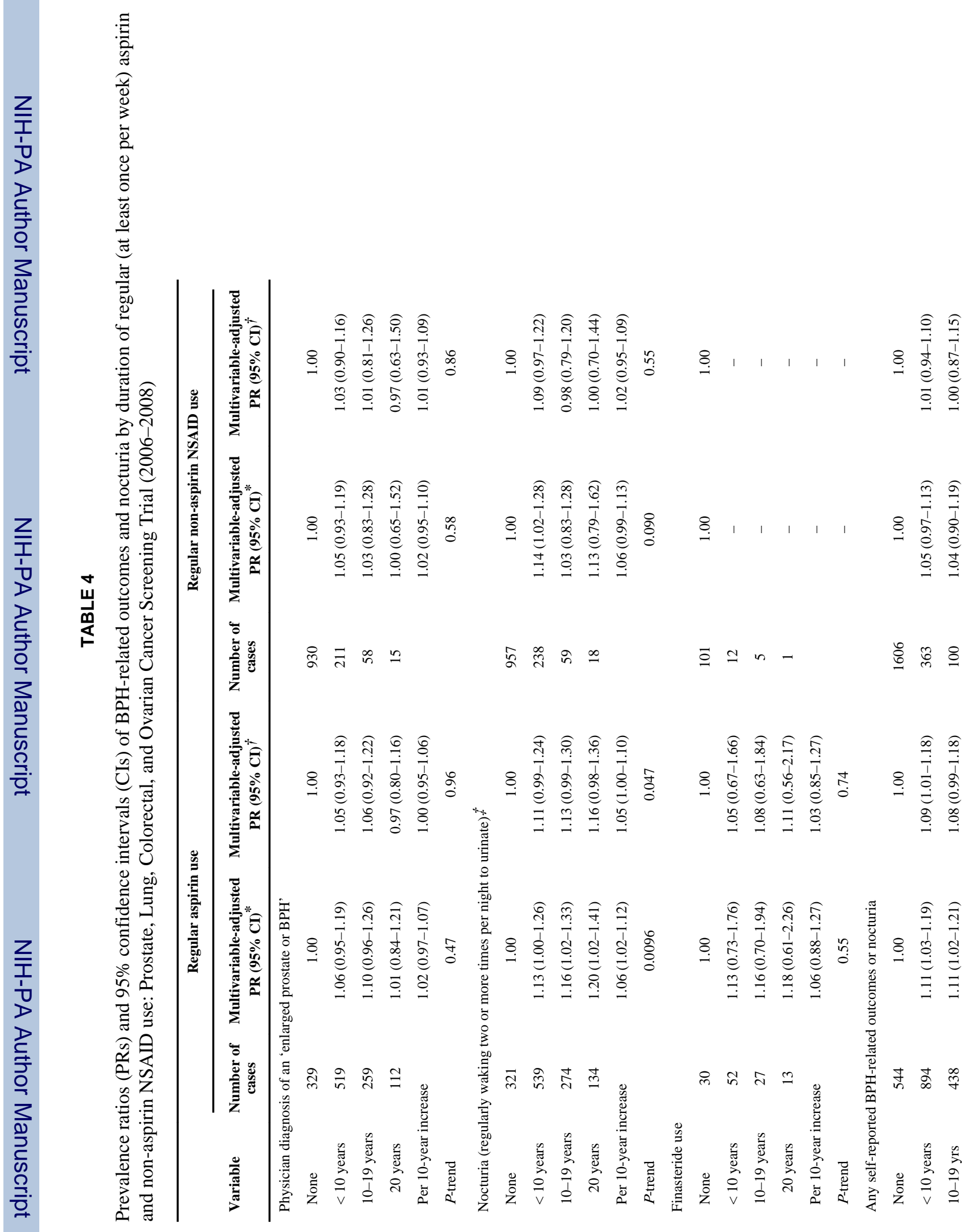

BJU Int. Author manuscript; available in PMC 2013 October 01. 


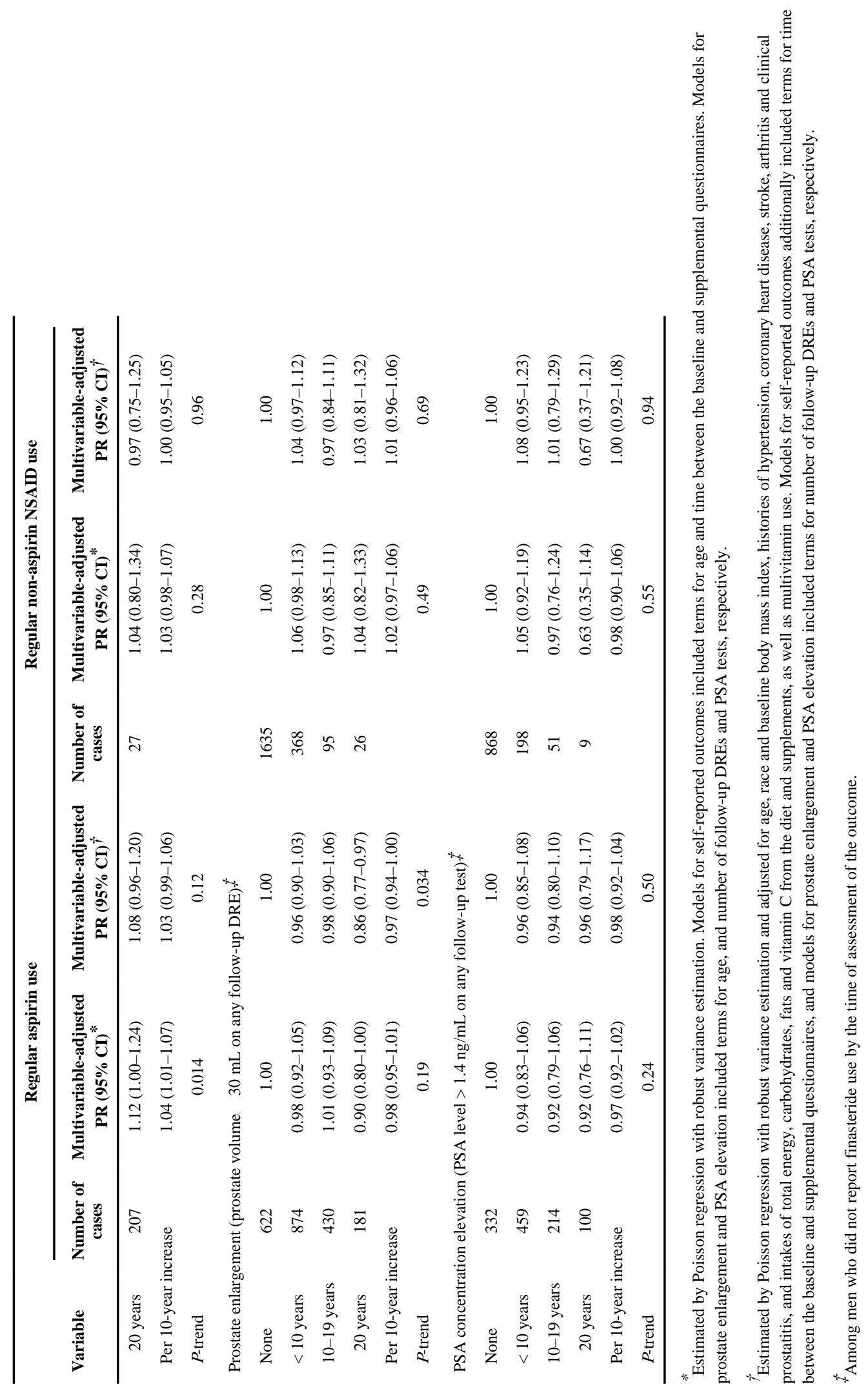




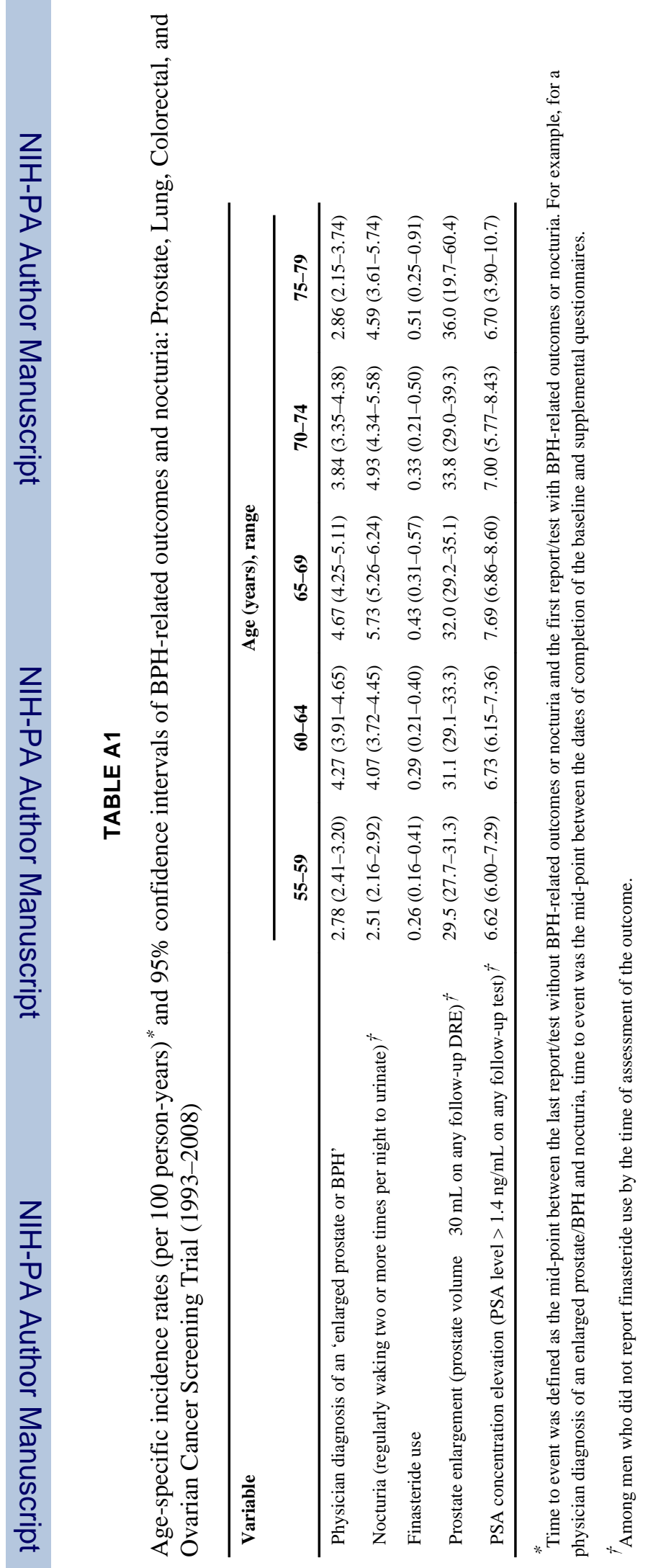

BJU Int. Author manuscript; available in PMC 2013 October 01. 